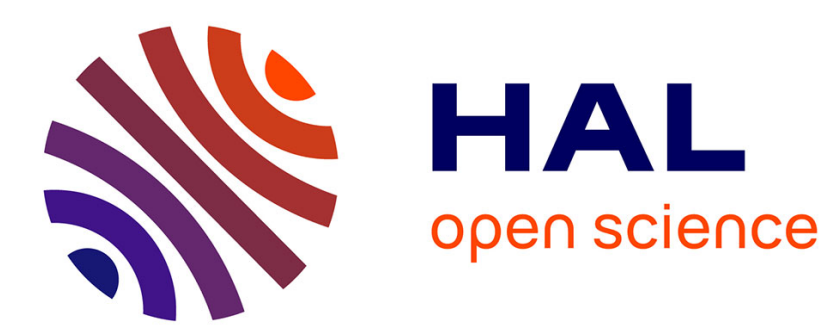

\title{
Non-divisible point on a two-parameter family of elliptic curves
}

Valentin Petit

\section{To cite this version:}

Valentin Petit. Non-divisible point on a two-parameter family of elliptic curves. 2021. hal$03115452 \mathrm{v} 2$

\section{HAL Id: hal-03115452 \\ https://hal.science/hal-03115452v2}

Preprint submitted on 19 Aug 2021

HAL is a multi-disciplinary open access archive for the deposit and dissemination of scientific research documents, whether they are published or not. The documents may come from teaching and research institutions in France or abroad, or from public or private research centers.
L'archive ouverte pluridisciplinaire HAL, est destinée au dépôt et à la diffusion de documents scientifiques de niveau recherche, publiés ou non, émanant des établissements d'enseignement et de recherche français ou étrangers, des laboratoires publics ou privés. 


\title{
Non-divisible point on a two-parameter family of elliptic curves
}

\author{
Valentin Petit
}

\begin{abstract}
Let $n$ be a positive integer and $t$ be a non-zero integer. We consider the two-parameter family of elliptic curves over $\mathbb{Q}$ given by

$$
\mathcal{E}_{n}(t): y^{2}=x^{3}+t x^{2}-n^{2}\left(t+3 n^{2}\right) x+n^{6} .
$$

We prove a result of non-divisibility of the point $\left(0, n^{3}\right) \in \mathcal{E}_{n}(t)(\mathbb{Q})$ whenever $t$ is sufficiently large compared to $n$ and $t^{2}+3 n^{2} t+9 n^{4}$ is squarefree. Our work extends to this family of elliptic curves a previous study of Duquesne mainly stated for $n=1$ and $t>0$.
\end{abstract}

MSC 2020: 11G05, 11G50.

Keywords: Elliptic curves, Integral points, Heights.

\section{Introduction}

We are concerned with proving the non-divisibility of a point on a family of elliptic curves defined over $\mathbb{Q}$ with two integer parameters. This family generalizes Washington's family [13] which is connected to simplest cubic fields. Let $n$ be a positive integer. We consider the elliptic curve over $\mathbb{Q}(T)$ (which can be seen as an elliptic surface over $\mathbb{P}^{1}(\mathbb{Q})$ ) given by

$$
\mathcal{E}_{n}: y^{2}=x^{3}+T x^{2}-n^{2}\left(T+3 n^{2}\right) x+n^{6} .
$$

It is a special case of an elliptic surface studied by Bettin, David and Delaunay [2]. The case $n=1$ is precisely Washington's family studied by Washington [13] and Duquesne [6]. In [2] the authors obtain a formula for the average root number of the elliptic curves obtained by specializing $\mathcal{E}_{n}$ at $T=t \in \mathbb{Q}$. We denote by $\mathcal{E}_{n}(t)$ this specialization.

Another elliptic curve over $\mathbb{Q}(T)$ derived from their family is

$$
\mathcal{F}_{n}: y^{2}=x^{3}+T x^{2}+n^{2}\left(T-3 n^{2}\right) x-n^{6} .
$$


Both elliptic curves $\mathcal{E}_{n}$ and $\mathcal{F}_{n}$ are related. Indeed, for all positive integers $n$ and all integers $t$, the curves $\mathcal{E}_{n}\left(t-3 n^{2}\right)$ and $\mathcal{F}_{n}(t)$ are isomorphic over $\mathbb{Q}$. We will focus on the curve $\mathcal{E}_{n}$.

Let $t \in \mathbb{Z}$ Theorem 5.7 of Duquesne in [6] asserts that if $n=1, t$ is positive and $t^{2}+3 t+9$ is squarefree, then the point $(0,1)$ is not divisible on $E_{1}(t)$. We generalize the result as follows for the integral point $\left(0, n^{3}\right)$.

Theorem 1. Let $n$ be a positive integer. Suppose that $t \geq \max \left(100 n^{2}, n^{4}\right)$ or $t \leq \min \left(-100 n^{2},-2 n^{4}\right)$, and $t^{2}+3 n^{2} t+9 n^{4}$ is squarefree. Then the point $\left(0, n^{3}\right)$ is not divisible on $\mathcal{E}_{n}(t)$.

As far as we know, there exists no similar statement for other twoparameter families of elliptic curves over $\mathbb{Q}$.

A consequence of Theorem 1 is that the point $\left(0, n^{3}\right)$ can be taken as an element of a system of generator for $E_{n}(t)(\mathbb{Q})$ when $t$ is sufficiently large. When the rank of $\mathcal{E}_{n}(t)$ is one, it is then possible to compute the analytic order of the Tate-Shafarevich group by using the BSD conjecture as in [5]. In particular, we note that except when $n=1$, the root number is not constant and not equidistributed which motivates the study of this type of family ([2]). For example when $n=2$, the average root number is $-\frac{1}{2}$, which means $75 \%$ of specializations have odd rank under the parity conjecture.

For all positive integer $n$, the elliptic curve $\mathcal{E}_{n}$ has rank one and the point $\left(0, n^{3}\right)$ has infinite order in $\mathcal{E}_{n}(\mathbb{Q}(T))([2]$, Theorem 1$)$. Silverman specialization Theorem on elliptic surfaces in [8] has the following consequence. Since the specialization map $\sigma_{t}: \mathcal{E}_{n} \rightarrow \mathcal{E}_{n}(t)$ is injective for all but only finitely many $t \in \mathbb{P}^{1}(\mathbb{Q})$, Theorem 1 implies that the point $\left(0, n^{3}\right)$ is a generator of $\mathcal{E}_{n}$. As regards the non-divisibility (see [8]) of the image of $\mathcal{E}_{n}$ by the specialization map, Theorem 2 in [8] asserts that the set of $t \in \mathbb{Z}$ such that $\sigma_{t}\left(\mathcal{E}_{n}\right)$ is non-divisible has density one. Our theorem holds provided that $t^{2}+3 n^{2} t+9 n^{4}$ is squarefree: it represents a set of $t \in \mathbb{Z}$ of density less than one, which gives a less strong result than predicted by Theorem 2 in [8] but is completely explicit. Furthermore, for all positive integer $n$, there exists a counterexample to non-divisibility when $t^{2}+3 n^{2} t+9 n^{4}$ is not squarefree. If $t=5 n^{2}$, we have $\left(0, n^{3}\right)=3\left(-4 n^{2}, 7 n^{3}\right)$ which shows that the squarefree hypothesis cannot be removed from Theorem 1 . However, note that we have not found another example where the point $\left(0, n^{3}\right)$ is the multiple of some point.

The strategy for proving Theorem 1 is similar to Duquesne's with an additional specific and careful treatment of the two parameters (we also notice that a small issue seems to occur in Section 5D of [6] during the computation of the local contribution of the height, which we can correct here). Suppose that there exist an integer $k \geq 2$ and a point $P \in \mathcal{E}_{n}(t)(\mathbb{Q})$ such that 
$k P=\left(0, n^{3}\right)$. The main idea is to minimize such an integer $k$. The strategy is to find a lower bound for the canonical height $\widehat{h}(P)$ of $P$, an upper bound for $\widehat{h}\left(\left(0, n^{3}\right)\right)$ (Section 4$)$, and to obtain a contradiction. To do this, we split the canonical height into local contributions (Section 4) and approximate the periods of $\mathcal{E}_{n}(t)$ (Section 3$)$.

\section{Generalities about the family}

Let $n$ be a positive integer and $t$ a non-zero integer. We consider the elliptic curve

$$
E: y^{2}=x^{3}+t x^{2}-n^{2}\left(t+3 n^{2}\right) x+n^{6},
$$

which was denoted by $\mathcal{E}_{n}(t)$ in the introduction. We set $\delta=t^{2}+3 n^{2} t+9 n^{4}$. The discriminant and $j$-invariant of the elliptic curve $E$ are given by

$$
\begin{aligned}
\Delta & =16 n^{4} \delta^{2}, \\
j & =\frac{256}{n^{4}} \delta, \\
c_{4} & =16 \delta .
\end{aligned}
$$

Let $f(x)$ be the polynomial

$$
f(x)=x^{3}+t x^{2}-n^{2}\left(t+3 n^{2}\right) x+n^{6} .
$$

Its discriminant is $n^{4} \delta^{2}$, which is positive, so $f$ has three real roots denoted by $\alpha_{1}<\alpha_{2}<\alpha_{3}$. They satisfy $\alpha_{1}<0<\alpha_{2}<n^{2}<\alpha_{3}$. The polynomial $f$ is irreducible over $\mathbb{Q}$ if $\delta$ is squarefree. Indeed, it suffices to see that the polynomial $h(x)=27 f\left(\frac{x-t}{3}\right)=x^{3}+3 \delta x+\delta\left(2 t+3 n^{2}\right)$ is irreducible over $\mathbb{Q}$ by Eisenstein's criterion.

We denote by $E_{0}(\mathbb{R})$ the connected component of the identity element and by $E(\mathbb{R})-E_{0}(\mathbb{R})$ the bounded connected component of $E(\mathbb{R})$. Recall that $E_{0}(\mathbb{R})$ is a subgroup of $E(\mathbb{R})$ and that the sum of two points in $E(\mathbb{R})-E_{0}(\mathbb{R})$ lies in $E_{0}(\mathbb{R})$. The integral point $\left(0, n^{3}\right)$ belongs to $E(\mathbb{Q})$ for all $t$ and $n$. More precisely, the fact that $\alpha_{2}>0$ implies that the point $\left(0, n^{3}\right)$ belongs to $E(\mathbb{Q})-E_{0}(\mathbb{Q})$ for all $t \in \mathbb{Z}_{\neq 0}$ and all positive integer $n$.

We note that $\left(0, n^{3}\right)$ is not a torsion point of $E$ when $\delta$ is squarefree. Indeed, since $\left(0, n^{3}\right) \in E(\mathbb{Q})-E_{0}(\mathbb{Q})$, the order of $\left(0, n^{3}\right)$ cannot be odd if it is finite. Moreover, since the polynomial $f(x)$ is irreducible over $\mathbb{Q}$ with the assumption $\delta$ squarefree, there is no torsion point of even order. Hence the point $\left(0, n^{3}\right)$ has infinite order.

Throughout the article we assume that $\delta$ is squarefree, which implies that $n$ and $t$ are coprime. This condition will play a key role later. Moreover 
we need (3) to be a minimal Weierstrass equation for $E$ which, by Tate's algorithm [11, IV.9], occurs if we assume also that $t$ is not congruent to 1 modulo 4 when $4 \mid n$. The minimal Weierstrass equation will be necessary to compute the non-archimedean local contributions.

For the case $4 \mid n$ and $t \equiv 1[4]$, write $n=4 m$ and $t=4 k+1$ with $m \in \mathbb{Z}_{>0}$ and $k \in \mathbb{Z}$. The elliptic curve $E$ is then isomorphic over $\mathbb{Q}$ to the curve

$$
E^{\prime}: y^{2}+x y=x^{3}+k x^{2}-m^{2}\left(4 k+1-48 m^{2}\right) x+64 m^{6} .
$$

The curve $E^{\prime}$ is connected to $E$ by the change of variables $x=4 x^{\prime}, y=$ $8 y^{\prime}+x^{\prime}$. Note that (4) is a minimal model for $E^{\prime}$. The change of variables maps the point $\left(0, n^{3}\right) \in E(\mathbb{Q})$ to the point $\left(0,8 m^{3}\right) \in E^{\prime}(\mathbb{Q})$. Therefore, if we want to prove the non-divisibility of $\left(0, n^{3}\right)$ on $E(\mathbb{Q})$, it suffices to prove the non-divisibility of $\left(0,8 \mathrm{~m}^{3}\right)$ on $E^{\prime}(\mathbb{Q})$.

\section{Approximation of periods}

The goal of this section is to approximate the real period $\omega_{1}$ and the imaginary period $\omega_{2}$ of $E$. In order to compute $\omega_{1}$ and $\omega_{2}$, the Weierstrass equation defining $E$ does not need to be minimal. Let $n$ be a fixed positive integer, the elliptic curve $E: y^{2}=x^{3}+t x^{2}-n^{2}\left(t+3 n^{2}\right) x+n^{6}$ is isomorphic over $\mathbb{Q}$ to the curve

$$
y^{2}=4 g(x)
$$

where $g(x)=x^{3}-\frac{1}{3} \delta x+\frac{1}{27}\left(2 t+3 n^{2}\right) \delta$. The real roots $e_{1}, e_{2}, e_{3}$ of $g$ are given by $e_{i}=\alpha_{i}+\frac{t}{3}$ for all $i \in\{1,2,3\}$ and the periods $\omega_{1}$ and $\omega_{2}$ of $E$ are (see $[3,7.3 .2])$

$$
\omega_{1}=\int_{e_{1}}^{e_{2}} \frac{d x}{\sqrt{g(x)}} \in \mathbb{R}, \quad \omega_{2}=-\int_{e_{2}}^{e_{3}} \frac{d x}{\sqrt{g(x)}} \in i \mathbb{R}
$$

\subsection{The case $t>0$}

When $t>0$, the real roots of $g$ satisfy $e_{1}<0<e_{2}<e_{3}$ because $g(0)>0$. A straightforward study of the function $g$ gives for $t \geq 3 n^{2}$,

$$
\begin{aligned}
-\frac{2}{3} t-n^{2}-2 \frac{n^{4}}{t} & \leq e_{1} \leq-\frac{2}{3} t-n^{2}-\frac{n^{4}}{t} \\
\frac{t}{3} & \leq e_{2} \leq \frac{t}{3}+\frac{n^{4}}{t} \\
\frac{t}{3}+n^{2} & \leq e_{3} \leq \frac{t}{3}+n^{2}+\frac{n^{4}}{t}
\end{aligned}
$$


Lemma 2. Let $n$ be a fixed positive integer. As $t \rightarrow+\infty$, we have $\frac{\omega_{2}}{i} \sim \frac{\pi}{\sqrt{t}}$. Moreover for $t \geq 100 n^{2}$, we have

$$
\frac{3.11}{\sqrt{t}} \leq \frac{\omega_{2}}{i} \leq \frac{3.15}{\sqrt{t}}
$$

Proof. We note that $\frac{\omega_{2}}{i}=\int_{e_{2}}^{e_{3}} \frac{d x}{\sqrt{\left(x-e_{1}\right)\left(x-e_{2}\right)\left(e_{3}-x\right)}}$. If $x \in\left[e_{2}, e_{3}\right]$, we have $t+n^{2}+\frac{n^{4}}{t} \leq x-e_{1} \leq t+2 n^{2}+\frac{3 n^{4}}{t}$ by $(5)$. So

$$
\frac{1}{\sqrt{t+2 n^{2}+\frac{3 n^{4}}{t}}} \leq \frac{1}{\sqrt{x-e_{1}}} \leq \frac{1}{\sqrt{t+n^{2}+\frac{n^{4}}{t}}}
$$

and then

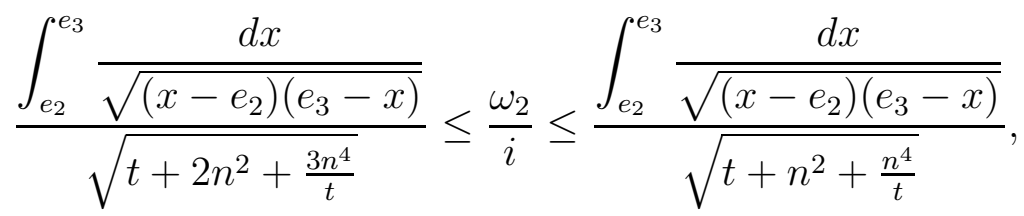

where $\int_{e_{2}}^{e_{3}} \frac{d x}{\sqrt{\left(x-e_{2}\right)\left(e_{3}-x\right)}}=\pi$. Moreover, as $t \rightarrow+\infty$ the left-hand side and the right-hand side of $(6)$ are both equivalent to $\frac{\pi}{\sqrt{t}}$. Finally, when $t \geq 100 n^{2}$, we derive from $(6)$

$$
\frac{3.11}{\sqrt{t}} \leq \frac{\omega_{2}}{i} \leq \frac{3.15}{\sqrt{t}} .
$$

Lemma 3. For $t \geq 100 n^{2}$, we have

$$
\frac{1.88+0.99 \log \left(\frac{t}{n^{2}}\right)}{\sqrt{t}} \leq \omega_{1} \leq \frac{5.35+1.23 \log \left(\frac{t}{n^{2}}\right)}{\sqrt{t}} .
$$

Proof. We split the integral into two parts

$$
\omega_{1}^{-}=\int_{e_{1}}^{0} \frac{d x}{\sqrt{\left(x-e_{1}\right)\left(e_{2}-x\right)\left(e_{3}-x\right)}}, \omega_{1}^{+}=\int_{0}^{e_{2}} \frac{d x}{\sqrt{\left(x-e_{1}\right)\left(e_{2}-x\right)\left(e_{3}-x\right)}} .
$$


First we consider $\omega_{1}^{-}$. If $x \in\left[e_{1}, 0\right]$, we have $\frac{t}{3} \leq e_{2}-x \leq t+n^{2}+\frac{3 n^{4}}{t}$ and $\frac{t}{3}+n^{2} \leq e_{3}-x \leq t+2 n^{2}+\frac{3 n^{4}}{t}$ by (5). So we get the lower bound

$$
\begin{aligned}
\omega_{1}^{-} \sqrt{\left(t+n^{2}+\frac{3 n^{4}}{t}\right)\left(t+2 n^{2}+\frac{3 n^{4}}{t}\right)} & \geq \int_{e_{1}}^{0} \frac{d x}{\sqrt{x-e_{1}}} \\
& \geq 2 \sqrt{-e_{1}} \\
& \geq 2 \sqrt{\frac{2}{3} t+n^{2}+\frac{n^{4}}{t}}
\end{aligned}
$$

Furthermore using (5), we get the upper bound

$$
\omega_{1}^{-} \leq \frac{\int_{e_{1}}^{0} \frac{d x}{\sqrt{x-e_{1}}}}{\sqrt{\frac{t}{3}\left(\frac{t}{3}+n^{2}\right)}} \leq \frac{2 \sqrt{-e_{1}}}{\sqrt{\frac{t}{3}\left(\frac{t}{3}+n^{2}\right)}} \leq \frac{6}{\sqrt{t}} \sqrt{\frac{2}{3}+\frac{n^{2}}{t}+\frac{2 n^{4}}{t^{2}}} .
$$

Thus when $t \geq 100 n^{2}$, we obtain

$$
\frac{1.60}{\sqrt{t}} \leq \omega_{1}^{-} \leq \frac{4.94}{\sqrt{t}}
$$

Now we consider $\omega_{1}^{+}$. If $x \in\left[0, e_{2}\right]$, we have $\frac{2}{3} t+n^{2}+\frac{n^{4}}{t} \leq x-e_{1} \leq t+n^{2}+\frac{n^{4}}{t}$ by (5). So we have

$$
\frac{J}{\sqrt{t+n^{2}+\frac{3 n^{4}}{t}}} \leq \omega_{1}^{+} \leq \frac{J}{\sqrt{\frac{2}{3} t+n^{2}+\frac{n^{4}}{t}}}
$$

with

$$
J=\int_{0}^{e_{2}} \frac{d x}{\sqrt{\left(e_{2}-x\right)\left(e_{3}-x\right)}}=\log \left(\frac{\sqrt{e_{3}}+\sqrt{e_{2}}}{\sqrt{e_{3}}-\sqrt{e_{2}}}\right) .
$$

Moreover by (5), we have

$$
\frac{\frac{4}{3} t+n^{2}}{n^{2}+\frac{n^{4}}{t}} \leq \frac{\sqrt{e_{3}}+\sqrt{e_{2}}}{\sqrt{e_{3}}-\sqrt{e_{2}}} \leq \frac{\frac{2}{3} t+n^{2}+\frac{2 n^{4}}{t}+2 \sqrt{\left(\frac{t}{3}+n^{2}+\frac{n^{4}}{t}\right)\left(\frac{t}{3}+\frac{n^{4}}{t}\right)}}{n^{2}-\frac{n^{4}}{t}}
$$

which implies

$$
\frac{\frac{4}{3} t+n^{2}}{n^{2}+\frac{n^{4}}{t}} \leq \frac{\sqrt{e_{3}}+\sqrt{e_{2}}}{\sqrt{e_{3}}-\sqrt{e_{2}}} \leq \frac{\frac{4}{3} t+3 n^{2}+\frac{4 n^{4}}{t}}{n^{2}-\frac{n^{4}}{t}}
$$


So we get

$\frac{1}{\sqrt{t+n^{2}+\frac{3 n^{4}}{t}}} \log \left(\frac{\frac{4}{3} t+n^{2}}{n^{2}+\frac{n^{4}}{t}}\right) \leq \omega_{1}^{+} \leq \frac{1}{\sqrt{\frac{2}{3} t+n^{2}+\frac{n^{4}}{t}}} \log \left(\frac{\frac{4}{3} t+3 n^{2}+\frac{4 n^{4}}{t}}{n^{2}-\frac{n^{4}}{t}}\right)$.

Assume now that $t \geq 100 n^{2}$. We derive

$$
\begin{aligned}
\omega_{1}^{+} & \leq \frac{1}{\sqrt{\frac{2}{3} t+n^{2}+\frac{n^{4}}{t}}}\left(\log (t)+\log \left(\frac{4}{3}\right)+\log \left(1+\frac{3 n^{2}}{t}+\frac{4 n^{4}}{t^{2}}\right)-\log \left(n^{2}-\frac{n^{4}}{t}\right)\right) \\
& \leq \frac{0.41+1.23 \log \left(\frac{t}{n^{2}}\right)}{\sqrt{t}}
\end{aligned}
$$

and

$$
\omega_{1}^{+} \geq \frac{1}{\sqrt{t+n^{2}+\frac{3 n^{4}}{t}}} \log \left(\frac{4 t}{3\left(n^{2}+\frac{n^{4}}{t}\right)}\right) \geq \frac{0.28+0.99 \log \left(\frac{t}{n^{2}}\right)}{\sqrt{t}} .
$$

Finally we obtain the desired conclusion.

Remark. A numerical analysis suggests that $\omega_{1}$ should be equivalent to $\frac{\log \left(\frac{t}{n^{2}}\right)}{\sqrt{t}}$ as $t \rightarrow+\infty$.

\subsection{The case $t<0$}

Once again we use the fact that $E$ is isomorphic to the curve $y^{2}=4 g(x)$. A straightforward study of the function $g$ gives the following estimates when $t \leq-3 n^{2}:$

$$
\begin{aligned}
\frac{t}{3}+\frac{2 n^{4}}{t} & \leq e_{1} \leq \frac{t}{3}+\frac{n^{4}}{t} \\
\frac{t}{3}+n^{2}+\frac{2 n^{4}}{t} & \leq e_{2} \leq \frac{t}{3}+n^{2}+\frac{n^{4}}{t} \\
-\frac{2 t}{3}-n^{2} & \leq e_{3} \leq-\frac{2 t}{3} .
\end{aligned}
$$

Lemma 4. As $t \rightarrow-\infty$, we have $\omega_{1} \sim \frac{\pi}{\sqrt{|t|}}$. Moreover, for $t \leq-100 n^{2}$ we have

$$
\frac{3.14}{\sqrt{|t|}} \leq \omega_{1} \leq \frac{3.15}{\sqrt{|t|}}
$$


Proof. If $x \in\left[e_{1}, e_{2}\right]$, then $|t|-2 n^{2}+\frac{n^{4}}{|t|} \leq e_{3}-x \leq|t|+\frac{2 n^{4}}{|t|}$ by (7). So

$$
\frac{1}{\sqrt{|t|+\frac{2 n^{4}}{|t|}}} \leq \frac{1}{\sqrt{e_{3}-x}} \leq \frac{1}{\left|\sqrt{|t|}-\frac{n^{2}}{\sqrt{|t|}}\right|}
$$

We obtain

$$
\frac{J}{\sqrt{|t|+\frac{2 n^{4}}{|t|}}} \leq \omega_{1} \leq \frac{J}{\left|\sqrt{|t|}-\frac{n^{2}}{\sqrt{|t|}}\right|}
$$

with

$$
J=\int_{e_{1}}^{e_{2}} \frac{d x}{\sqrt{\left(x-e_{1}\right)\left(e_{2}-x\right)}}=\pi .
$$

Since $\frac{\pi}{\sqrt{|t|+\frac{2 n^{4}}{|t|}}}$ and $\frac{\pi}{\sqrt{|t|}-\frac{n^{2}}{\sqrt{|t|}}}$ are both equivalent to $\frac{\pi}{\sqrt{|t|}}$ as $t \rightarrow-\infty$, we deduce $\omega_{1} \underset{t \rightarrow-\infty}{\sim} \frac{\pi}{\sqrt{|t|}}$. Furthermore if $t \leq-100 n^{2}$, we get

$$
\frac{3.14}{\sqrt{|t|}} \leq \omega_{1} \leq \frac{3.15}{\sqrt{|t|}}
$$

Lemma 5. If $t \leq-100 n^{2}$, we have

$$
\frac{\omega_{2}}{i} \geq \frac{0.39+\log \left(\frac{|t|}{n^{2}}\right)}{\sqrt{|t|}} .
$$

Proof. Note that $e_{2}<0<e_{3}$ as $g(0)<0$ for $t \leq 100 n^{2}$. To approximate $\omega_{2}$, we need to split the integral into two parts:

$$
\frac{\omega_{2}}{i}=\underbrace{\int_{e_{2}}^{0} \frac{d x}{\sqrt{\left(x-e_{1}\right)\left(x-e_{2}\right)\left(e_{3}-x\right)}}}_{W^{-}}+\underbrace{\int_{0}^{e_{3}} \frac{d x}{\sqrt{\left(x-e_{1}\right)\left(x-e_{2}\right)\left(e_{3}-x\right)}}}_{W^{+}} .
$$

We begin with estimating $W^{-}$. If $x \in\left[e_{2}, 0\right]$, we get by $(7)$

$$
-\frac{2}{3} t-n^{2} \leq e_{3}-x \leq-t-n^{2}-\frac{2 n^{4}}{t}
$$

So

$$
\frac{L}{\sqrt{-t-n^{2}-\frac{2 n^{4}}{t}}} \leq W^{-} \leq \frac{L}{\sqrt{-\frac{2}{3} t-n^{2}}}
$$


with

$$
L=-\int_{0}^{e_{2}} \frac{d x}{\sqrt{\left(x-e_{1}\right)\left(x-e_{2}\right)}}=-\log \left(e_{2}-e_{1}\right)+\log \left(-e_{1}-e_{2}+\sqrt{e_{1} e_{2}}\right) .
$$

Moreover by (7), we have

$$
-\log \left(n^{2}-\frac{n^{4}}{t}\right)+\log \left(-\frac{2}{3} t-n^{2}-\frac{2 n^{4}}{t}\right) \leq L .
$$

For $t \leq-100 n^{2}$, we get

$$
\begin{aligned}
W^{-} \sqrt{-t-n^{2}-\frac{2 n^{4}}{t}} & \geq-\log \left(n^{2}-\frac{n^{4}}{t}\right)+\log \left(-\frac{2}{3} t-n^{2}-\frac{2 n^{4}}{t}\right) \\
& \geq-2 \log (n)-\log \left(1-\frac{1}{100}\right)+\log (|t|)+\log \left(\frac{2}{3}-\frac{1}{100}\right),
\end{aligned}
$$

from which we derive

$$
W^{-} \geq \frac{\log \left(\frac{|t|}{n^{2}}\right)-0.42}{\sqrt{|t|}} .
$$

If $x \in\left[0, e_{3}\right]$, we obtain by $(7)$

$$
\begin{gathered}
-\frac{t}{3}-\frac{n^{4}}{t} \leq x-e_{1} \leq-t-\frac{2 n^{4}}{t} \\
-\frac{t}{3}-n^{2}-\frac{n^{4}}{t} \leq x-e_{2} \leq-t-n^{2}-\frac{2 n^{4}}{t} .
\end{gathered}
$$

Thus

$$
W^{+} \sqrt{\left(-t-\frac{2 n^{4}}{t}\right)\left(-t-n^{2}-\frac{n^{4}}{t}\right)} \geq 2 \sqrt{e_{3}},
$$

from which we derive

$$
W^{+} \geq \frac{\sqrt{-\frac{2}{3} t-n^{2}}}{\sqrt{\left(-t-\frac{2 n^{4}}{t}\right)\left(-t-n^{2}-\frac{n^{4}}{t}\right)}} \geq \frac{\sqrt{|t|} \sqrt{\frac{2}{3}-\frac{n^{2}}{|t|}}}{|t| \sqrt{\left(1+\frac{2 n^{4}}{|t|^{2}}\right)^{2}}} .
$$

So we obtain, for $t \leq-100 n^{2}$

$$
W^{+} \geq \frac{0.81}{\sqrt{|t|}}
$$

Finally by adding both inequalities (8) and (9), we have

$$
\frac{\omega_{2}}{i} \geq \frac{0.39+\log \left(\frac{|t|}{n^{2}}\right)}{\sqrt{|t|}} \text {. }
$$

Remark. When $t<0$, we just give a lower bound for $\frac{\omega_{2}}{i}$ since no upper bound is required in order estimate the height. 


\section{Estimates on the heights}

We need to estimate the heights of some points in $E(\mathbb{Q})$. For this purpose, we will decompose the height as a sum of local contributions. Depending on the conventions, there are different ways to split the canonical height into a sum of local heights. However in this section, a special care was taken during the computations of local heights to make sure that their sum agrees with the definition of the canonical height.

\subsection{Lower bound for the height}

We want to prove that there does not exist a point $P=(\alpha, \beta) \in E(\mathbb{Q})$ and an integer $\ell \geq 2$ such that $\ell P=\left(0, n^{3}\right)$. The goal of this part is to find, if such a point exists, a lower bound for the canonical height of $P$. Since $\left(0, n^{3}\right) \in E(\mathbb{Q})-E_{0}(\mathbb{Q})$, the point $P$ has to belong to $E(\mathbb{Q})-E_{0}(\mathbb{Q})$ and $\ell$ must be odd (see Section 2).

Lemma 6. Let $F: y^{2}+a_{1} x y+a_{3} y=x^{3}+a_{2} x^{2}+a_{4} x+a_{6}$ be an elliptic curve over $\mathbb{Q}$ with $a_{i} \in \mathbb{Z}$ for all $i \in\{1,2,3,4,6\}$. Let $P \in F(\mathbb{Q})$ be a point of infinite order such that $m P$ is an integral point for some integer $m \geq 1$. Then $P$ is an integral point on $F$.

Proof. A proof of this lemma is established in [1]. Write $m P=(\alpha, \beta)$ with $\alpha, \beta \in \mathbb{Z}$ and $P=(x, y)$ with $x, y \in \mathbb{Q}$. Let $\psi_{m}, \phi_{m}$ be the $m^{\text {th }}$-division polynomials. Recall that $\phi_{m}$ is a monic polynomial in $\mathbb{Z}[X]$ of degree $m^{2}$ and $\psi_{m}^{2}$ is a polynomial in $\mathbb{Z}[X]$ of degree $m^{2}-1$ (for more details see [1], Section 2). We have

$$
\frac{\phi_{m}(x)}{\psi_{m}^{2}(x)}=\alpha .
$$

We get that $x$ is a root of the polynomial $\phi_{m}(X)-\alpha \psi_{m}^{2}(X)$. Since $\phi_{m}(X)-$ $\alpha \psi_{m}^{2}(X)$ is monic in $\mathbb{Z}[X]$ and $x \in \mathbb{Q}$, we deduce that $x \in \mathbb{Z}$ and then $y \in \mathbb{Z}$. Hence $P$ is an integral point.

Lemma 6 implies that if $\left(0, n^{3}\right)$ is the multiple of a rational point $P$ then $P \in E(\mathbb{Z})$. Moreover the point $\left(0, n^{3}\right)$ is singular modulo $p$ for all primes $p \mid n$. This implies that $P$ is singular modulo $p$ for all $p \mid n$ since the multiple of a non-singular point is always non-singular. If $p \mid n$, the only singular point modulo $p$ is $(0,0)$ so we have $p \mid \alpha$. This remark will be used in Lemmas 7, 8 and Proposition 11.

Lemma 7. Let $P=(\alpha, \beta) \in E(\mathbb{Z})-E_{0}(\mathbb{Z})$ be such that there exists $\ell \geq 2$ with $\ell P=\left(0, n^{3}\right)$. If $t \geq n^{4}$ then $|\beta| \geq n \sqrt{2 t}$. 
Proof. Assume that $t \geq n^{4}$ and $n \geq 2$. Recall that $\alpha_{1}, \alpha_{2}, \alpha_{3}$ are the real roots of the polynomial $f(x)=x^{3}+t x^{2}-n^{2}\left(t+3 n^{2}\right) x+n^{6}$ with $\alpha_{1}<0<\alpha_{2}<\alpha_{3}$. By studying the function $f$, we have for $t \geq n^{4}$

$$
-n^{2}-t-1<\alpha_{1}<-n^{2}-t \text { and } 0<\alpha_{2}<1 .
$$

Since $P \in E(\mathbb{Q})-E_{0}(\mathbb{Q})$, we have $\alpha_{1}<\alpha<\alpha_{2}$, and then $\alpha \in\left[-n^{2}-t,-1\right]$. Moreover since $p \mid \alpha$ for all primes $p \mid n$, we get $\alpha \leq-2$ and then

$$
\begin{aligned}
f(\alpha) & \geq \min \left(f\left(-n^{2}-t\right), f(-2)\right) \\
& \geq \min \left(2 n^{4} t+3 n^{6},\left(2 n^{2}+4\right) t+n^{6}+6 n^{4}-8\right) \\
& \geq 2 n^{2} t .
\end{aligned}
$$

Thus $|\beta| \geq n \sqrt{2 t}$. If $n=1$, we have

$$
f(\alpha) \geq \min (f(-t-1), f(-1))=2 t+3,
$$

hence $|\beta| \geq \sqrt{2 t+3} \geq \sqrt{2 t}$.

Lemma 8. Suppose that $t \leq-2 n^{4}$. Then

$$
|\beta| \geq \begin{cases}\sqrt{2|t|} & \text { if } n=2 \\ n \sqrt{|t|} & \text { if } n \geq 3\end{cases}
$$

Proof. We use an argument similar to the case $t \geq n^{4}$ (Lemma 7 ).

Remark. Assume $n=1$. If $t \leq-2$, the point $(0,1)$ is the only integral point in $E(\mathbb{Q})-E_{0}(\mathbb{Q})$ because $-1<\alpha_{1}<\alpha_{2}$. Hence $(0,1)$ is not divisible. If $t=-1$, it is easy to prove that this point is not divisible as well. Therefore when $t$ is negative, we can assume that $n \geq 2$.

In order to find a lower bound for the canonical height of an integral point of $E$, we use the decomposition of the canonical height into local contributions (see $[9$, Theorem 5.2], [4, 7.5.7]). Let $P=(\alpha, \beta) \in E(\mathbb{Q})$ be an integral point, we have

$$
\widehat{h}(P)=\sum_{p \leq \infty} \lambda_{p}(P),
$$

where the sum runs through the places of $\mathbb{Q}$. We recall the definitions of $\lambda_{p}$ for finite places $p$ in Definition/Proposition 9 and $\lambda_{\infty}$ in Definition/Proposition 12. For this, we set

$$
\begin{aligned}
& A=3 \alpha^{2}+2 t \alpha-n^{2}\left(t+3 n^{2}\right), \\
& B=2 \beta, \\
& C=3 \alpha^{4}+4 t \alpha^{3}-6 n^{2}\left(t+3 n^{2}\right) \alpha^{2}+12 n^{6} \alpha-n^{4}\left(t^{2}+2 n^{2} t+9 n^{4}\right), \\
& D=\operatorname{gcd}(A, B), \\
& c_{4}=16 \delta .
\end{aligned}
$$


Definition/Proposition 9. ([9, Theorem 5.2],[4, 7.5.6]) Let $p$ be a prime number. The local non-archimedean contribution $\lambda_{p}(P)$ is non-zero only if $p \mid D$. If $p \mid D$, we set $m_{p}=\min \left(\frac{v_{p}(\Delta)}{2}, v_{p}(B)\right)$ and the local contribution at $p$ is then given by

$$
\lambda_{p}(P)= \begin{cases}-\frac{m_{p}\left(v_{p}(\Delta)-m_{p}\right)}{2 v_{p}(\Delta)} \log (p) & \text { if } p \nmid c_{4}, \\ -\frac{v_{p}(B)}{3} \log (p) & \text { if } p \mid c_{4} \text { and } v_{p}(C) \geq 3 v_{p}(B), \\ -\frac{v_{p}(C)}{8} \log (p) & \text { otherwise, }\end{cases}
$$

where $v_{p}$ is the $p$-adic valuation.

Lemma 10. Assume $4 \nmid n$ or $t \not \equiv 1[4]$. If $p \nmid 2 n$, then $\lambda_{p}(P)=0$.

Proof. Let $p \mid D$. We assume that $p \nmid n$ and $p \neq 2$. First we have $4 A^{2}=$ $B^{2}(9 \alpha+3 t)+4 \delta\left(\alpha^{2}-n^{2} \alpha+n^{4}\right)$. Since $\delta$ is squarefree, we obtain that $p \mid\left(\alpha^{2}-n^{2} \alpha+n^{4}\right)$. We also have

$$
B^{2}=4\left(\alpha^{2}-n^{2} \alpha+n^{4}\right)\left(\alpha+t+n^{2}\right)-4 n^{2}(3 \alpha+t)
$$

so $p \mid(3 \alpha+t)$. Furthermore $p$ divides the resultant of $A$ and $B^{2}$, viewed as polynomials in $\mathbb{Z}[\alpha]$, which is equal to $\Delta=16 n^{4} \delta^{2}$. Therefore $p \mid \delta$. Moreover,

$$
27 B^{2}=4(3 \alpha+t)^{3}-4 \delta\left(9 \alpha+t-3 n^{2}\right),
$$

which implies that $p \mid\left(9 \alpha+t-3 n^{2}\right)$. Since $\left(9 \alpha+t-3 n^{2}\right)=3(3 \alpha+t)-$ $\left(2 t+3 n^{2}\right)$, we obtain that $p \mid\left(2 t+3 n^{2}\right)$. But $4 \delta=\left(2 t+3 n^{2}\right)^{2}+27 n^{4}$. We get $p=3$ hence $\delta$ is not squarefree, which is a contradiction.

Proposition 11. Let $P=(\alpha, \beta)$ be an integral point on $E$ such that $\ell P=$ $\left(0, n^{3}\right)$ for some integer $\ell \geq 1$. Then the following inequality holds

$$
\sum_{p<\infty} \lambda_{p}(P) \geq-\frac{1}{2} \log (n)-\frac{1}{3} \log (2) .
$$

Proof. By Lemma 10, it suffices to compute the local contributions at primes $p \mid 2 n$. For $p \mid n$, we first assume $p \neq 2$. Let $m=v_{p}(n)$. Since $\delta$ is squarefree, we have $p \nmid c_{4}$ and $v_{p}(\Delta)=4 m$. Put $m_{p}=\min \left(2 m, v_{p}(B)\right)$. By Definition/Proposition 9 , the local contribution of $P$ is then given by

$$
\lambda_{p}(P)=-\frac{m_{p}\left(4 m-m_{p}\right)}{8 m} \log (p)
$$


We easily conclude that

$$
\lambda_{p}(P) \geq-\frac{m}{2} \log (p) \geq-\frac{1}{2} \log \left(p^{m}\right) .
$$

Now we want to compute the 2 -local contribution $\lambda_{2}(P)$. Since $2 \mid c_{4}$, it is given by

$$
\lambda_{2}(P)= \begin{cases}-\frac{v_{2}(B)}{3} \log (2) & \text { if } v_{2}(C) \geq 3 v_{2}(B), \\ -\frac{v_{2}(C)}{8} \log (2) & \text { otherwise. }\end{cases}
$$

If $2 \nmid n$ then $v_{2}(B)=1$, so $\lambda_{2}(P) \geq-\frac{1}{3} \log (2)$.

Suppose now that $2 \mid n$ and $m=v_{2}(n)$.

If $m \in\{1,2\}$ it is easy to check that $\lambda_{2}(P) \geq-\frac{1}{2} \log \left(2^{m}\right)-\frac{1}{3} \log (2)$. So we may assume that $m \geq 3$.

If $v_{2}(\alpha)<m$ then $v_{2}(B)=1+v_{2}(\alpha)$; in that case we have $\lambda_{2}(P) \geq \frac{-m}{3} \log (2)$ if $v_{2}(C) \geq 3 v_{2}(B)$, else $\lambda_{2}(P) \geq-\frac{m}{2} \log (2)$.

If $m \leq v_{2}(\alpha)<\frac{4 m-2}{3}$ then $v_{2}(B)=1+v_{2}(\alpha)$ and $v_{2}(C)=3 v_{2}(\alpha)+2$; in that case we have $\lambda_{2}(P)=-\frac{3 v_{2}(\alpha)+2}{8} \log (2) \geq-\frac{1}{2} \log \left(2^{m}\right)$.

If $v_{2}(\alpha)=\frac{4 m-2}{3}$ which is possible only if $m \equiv 2[3]$ then $v_{2}(C)>4 m$ and $v_{2}(B)=\frac{4 m+1}{3}$. In that case we have $\lambda_{2}(P)=-\frac{4 m+1}{9} \log (2) \geq$ $-\frac{m}{2} \log (2)$.

If $v_{2}(\alpha)>\frac{4 m-2}{3}$, then $v_{2}(C)=4 m$ and $3 v_{2}(B)>4 m$; in that case we have $\lambda_{2}(P) \geq-\frac{1}{2} \log \left(2^{m}\right)$.

Finally we conclude by summing all local non-archimedean contributions.

Now we study the local archimedean contribution.

Definition/Proposition 12. ([4, 7.5.7]) Let $P=(\alpha, \beta) \in E(\mathbb{Q})$. Let $z$ be the elliptic logarithm of $P$. Let $\mu=\frac{2 \pi}{\omega_{1}}, s=\mu \operatorname{Re}(z), q=\exp \left(\frac{2 i \pi \omega_{2}}{\omega_{1}}\right)$, and

$$
\theta=\sum_{k=0}^{\infty} \sin ((2 k+1) s)(-1)^{k} q^{\frac{k(k+1)}{2}}
$$


Then the local archimedean contribution is given by

$$
\lambda_{\infty}(P)=\frac{1}{32} \log \left|\frac{\Delta}{q}\right|-\frac{1}{4} \log |\theta|+\frac{1}{8} \log \left|\frac{\alpha^{3}+\frac{b_{2}}{4} \alpha^{2}+\frac{b_{4}}{2} \alpha+\frac{b_{6}}{4}}{\mu}\right|
$$

where $b_{2}=a_{1}^{2}+4 a_{2}, b_{4}=a_{1} a_{3}+2 a_{4}, b_{6}=a_{3}^{2}+4 a_{6}$ and $a_{1}, a_{2}, a_{3}, a_{4}$ and $a_{6}$ are defined as in Lemma 6.

Proposition 13. Assume that $4 \nmid n$ or $t \not \equiv 1[4]$ and that $\ell P=\left(0, n^{3}\right)$, for some integer $\ell$. If $t \geq \max \left(100 n^{2}, n^{4}\right)$, we have

$$
\lambda_{\infty}(P) \geq \frac{13}{80} \log (t)+\frac{3}{8} \log (n)+0.30 .
$$

If $t \leq \min \left(-100 n^{2},-2 n^{4}\right)$, we have

$$
\lambda_{\infty}(P) \geq \frac{3}{16} \log (|t|)+\frac{3}{8} \log (n)+0.27 .
$$

Proof. We first note that

$$
|\theta| \leq \sum_{k=0}^{\infty} q^{\frac{k(k+1)}{2}} \leq \frac{1}{1-q}
$$

To find a lower bound for $\lambda_{\infty}(P)$, we need to find an upper bound for $q$. If $t \geq 100 n^{2}$, by Lemmas 2 and 3, we have

$$
\frac{2 i \pi \omega_{2}}{\omega_{1}} \leq \frac{-3.11 \times 2 \pi}{5.35+1.23 \log \left(\frac{t}{n^{2}}\right)} \leq \frac{-15.88}{\log \left(\frac{t}{n^{2}}\right)}
$$

thus

$$
q \leq \exp \left(\frac{-15.88}{\log \left(\frac{t}{n^{2}}\right)}\right) \leq 1-\frac{4.3}{\log \left(\frac{t}{n^{2}}\right)}
$$

On the one hand, by definition of $\Delta$ we have

$$
\frac{1}{32} \log (\Delta)=\frac{1}{32} \log \left(16 n^{4}\left(t^{2}+3 n^{2} t+9 n^{4}\right)^{2}\right) \geq \frac{1}{8} \log (t)+\frac{1}{8} \log (n)+\frac{1}{8} \log (2) .
$$

On the other hand, with (11) and (10) we get

$$
\begin{gathered}
\frac{1}{32} \log \left|\frac{1}{q}\right| \geq \frac{1}{32} \log \left(\exp \left(\frac{15.88}{\log \left(\frac{t}{n^{2}}\right)}\right)\right) \geq \frac{15.88}{32 \log \left(\frac{t}{n^{2}}\right)}, \\
-\frac{1}{4} \log |\theta| \geq-\frac{1}{4} \log \left|\frac{1}{1-q}\right| \geq \frac{1}{4} \log (4.3)-\frac{1}{4} \log \log \left(\frac{t}{n^{2}}\right),
\end{gathered}
$$


and by Lemma 3 ,

$$
\begin{aligned}
-\frac{1}{8} \log (\mu)=\frac{1}{8} \log \left(\frac{\omega_{1}}{2 \pi}\right) & \geq-\frac{1}{8} \log (2 \pi)+\frac{1}{8} \log \left(\frac{1.88+0.99 \log \left(\frac{t}{n^{2}}\right)}{\sqrt{t}}\right), \\
& \geq-\frac{1}{16} \log (t)-\frac{1}{8} \log (2 \pi)+\frac{1}{8} \log \log \left(\frac{t}{n^{2}}\right)+\frac{1}{8} \log (0.99) .
\end{aligned}
$$

Moreover, we have

$$
\frac{1}{8} \log (2)+\frac{1}{4} \log (4.3)-\frac{1}{8} \log (2 \pi)+\frac{1}{8} \log (0.99) \geq 0.22 .
$$

Finally using Definition/Proposition 12 and the fact that $\alpha^{3}+\frac{b_{2}}{4} \alpha^{2}+\frac{b_{4}}{2} \alpha+$ $\frac{b_{6}}{4}=\beta^{2}$, we obtain the lower bound for $\lambda_{\infty}(P)$ :

$$
\begin{aligned}
\lambda_{\infty}(P) \geq & \frac{1}{16} \log (t)-\frac{1}{8} \log \log \left(\frac{t}{n^{2}}\right)+\frac{15.88}{32 \log \left(\frac{t}{n^{2}}\right)} \\
& +\frac{1}{4} \log |\beta|+\frac{1}{8} \log (n)+0.22 .
\end{aligned}
$$

Moreover, by Lemma 7 , for $t \geq n^{4}$ we have $|\beta| \geq n \sqrt{2 t}$. If $t \geq \max \left(n^{4}, 100 n^{2}\right)$, we obtain

$$
\lambda_{\infty}(P) \geq \frac{13}{80} \log (t)+\frac{3}{8} \log (n)+0.30 .
$$

When $t$ is negative, we proceed similarly to find a lower bound to $\lambda_{\infty}(P)$. If $t \leq-100 n^{2}$, we have by Lemmas 4 and 5

$$
\frac{2 i \pi \omega_{2}}{\omega_{1}} \leq \frac{-2 \pi\left(0.39+\log \left(\frac{|t|}{n^{2}}\right)\right)}{3.15} \leq-\frac{2 \pi}{3.15} \log \left(\frac{|t|}{n^{2}}\right),
$$

and

$$
q \leq \exp \left(-\frac{2 \pi}{3.15} \log \left(\frac{|t|}{n^{2}}\right)\right) \leq 0.0002
$$

On the one hand, by definition of $\Delta$ we have

$$
\frac{1}{32} \log (\Delta) \geq \frac{1}{8} \log (|t|)+\frac{1}{8} \log (n)+\frac{1}{8} \log (2)+\frac{1}{32} \log \left(1-\frac{3}{100}\right)
$$

On the other hand, with (12) and (10) we get

$$
\begin{aligned}
& \frac{1}{32} \log \left|\frac{1}{q}\right| \geq \frac{1}{32} \log \exp \left(\frac{2 \pi}{3.15} \log \left(\frac{|t|}{n^{2}}\right)\right) \geq 0.28 \\
& -\frac{1}{4} \log |\theta| \geq-\frac{1}{4} \log \left|\frac{1}{1-q}\right| \geq-\frac{1}{4} \log \left(\frac{1}{1-0.0002}\right),
\end{aligned}
$$


and, by Lemma 4,

$$
\begin{aligned}
-\frac{1}{8} \log (\mu) & \geq-\frac{1}{8} \log (2 \pi)+\frac{1}{8} \log \left(\frac{3 \cdot 14}{\sqrt{|t|}}\right) \\
& \geq-\frac{1}{16} \log (|t|)-\frac{1}{8} \log (2 \pi)+\frac{1}{8} \log (3.14) .
\end{aligned}
$$

Moreover we have

$$
\frac{1}{8} \log (2)+\frac{1}{8} \log (3.14)-\frac{1}{8} \log (2 \pi)+\frac{1}{4} \log (1-0.0002)+0.28 \geq 0.27 .
$$

Then we obtain

$$
\lambda_{\infty}(P) \geq \frac{1}{16} \log (|t|)+\frac{1}{4} \log |\beta|+\frac{1}{8} \log (n)+0.27 .
$$

Moreover by Lemma 8 , for $t \leq-2 n^{4}$, we have $|\beta| \geq n \sqrt{|t|}$. If $t \leq \min \left(-2 n^{4},-100 n^{2}\right)$, we obtain

$$
\lambda_{\infty}(P) \geq \frac{3}{16} \log (|t|)+\frac{3}{8} \log (n)+0.27 .
$$

Theorem 14. Assume that $4 \nmid n$ or $\not \equiv 1[4]$. Let $P$ be an integral point on $E$ such that $\ell P=\left(0, n^{3}\right)$ for some integer $\ell \geq 2$. If $t \geq \max \left(100 n^{2}, n^{4}\right)$, we have

$$
\widehat{h}(P) \geq \frac{13}{80} \log (t)-\frac{1}{8} \log (n)+0.09 .
$$

If $t \leq \min \left(-100 n^{2},-n^{4}\right)$, we have

$$
\widehat{h}(P) \geq \frac{3}{16} \log (|t|)-\frac{1}{8} \log (n) .
$$

Proof. It suffices to sum the inequalities obtained in Proposition 11 and Proposition 13.

By similar arguments, we obtain the following statements for the case $t=4 k+1$ and $n=4 m$.

Proposition 15. If $P=(\alpha, \beta)$ is an integral point of $E^{\prime}$ such that $\ell P=$ $\left(0,8 \mathrm{~m}^{3}\right)$ for some integer $\ell \geq 1$, then the following inequality holds

$$
\sum_{p<\infty} \lambda_{p}(P) \geq-\frac{1}{2} \log (m) \geq-\frac{1}{2} \log (n)+\log (2) .
$$


Proposition 16. Let $P \in E^{\prime}(\mathbb{Q})$ be an integral point such that $\ell P=\left(0,8 m^{3}\right)$ for some integer $\ell \geq 2$. If $t \geq \max \left(n^{4}, 100 n^{2}\right)$, we have

$$
\lambda_{\infty}(P) \geq \frac{13}{80} \log (t)+\frac{3}{8} \log (n)-\frac{5}{8} \log (2)+0.04 .
$$

If $t \leq \min \left(-100 n^{2},-2 n^{4}\right)$, we have

$$
\lambda_{\infty}(P) \geq \frac{3}{16} \log (|t|)+\frac{3}{8} \log (n)-\frac{3}{4} \log (2)+0.10 .
$$

Again after summing the inequalities obtained in Propositions 15 and 16, we obtain the following statement.

Theorem 17. Let $P$ be an integral point on $E^{\prime}$ such that $\ell P=\left(0,8 m^{3}\right)$, for some integer $\ell \geq 2$. If $t \geq \max \left(100 n^{2}, n^{4}\right)$, we have

$$
\widehat{h}(P) \geq \frac{13}{80} \log (t)+\frac{3}{8} \log (n)+0.04 .
$$

If $t \leq \min \left(-100 n^{2},-2 n^{4}\right)$, we have

$$
\widehat{h}(P) \geq \frac{3}{16} \log (|t|)+\frac{1}{4} \log (n)+0.10 .
$$

\subsection{Upper bound for the height}

Proposition 18. If $|t| \geq 100 n^{2}$, we have the following inequality

$$
\widehat{h}\left(\left(0, n^{3}\right)\right) \leq \log (|t|)+1.57 \text {. }
$$

Proof. We use the result of Silverman [10, Theorem 1.1]. Let $P \in E(\mathbb{Q})$. We have

$$
\widehat{h}(P)-\frac{1}{2} h(P) \leq \frac{1}{12} h(\Delta)+\frac{1}{12} h_{\infty}(j)+\frac{1}{2} h_{\infty}\left(\frac{b_{2}}{12}\right)+\frac{1}{2} \log (2)+1.07
$$

where $h(P)=h(x(P))$ is the logarithmic height over $\mathbb{Q}, h_{\infty}(x)=\max (\log |x|, 0)$ and $b_{2}$ is defined as in Definition/Proposition 12. Since $h\left(\left(0, n^{3}\right)\right)=0$, in our case we have

$$
\begin{aligned}
\widehat{h}\left(\left(0, n^{3}\right)\right) \leq & \frac{1}{12} \log \left(16 n^{4}\left(t^{2}+3 n^{2} t+9 n^{4}\right)^{2}\right)+\frac{1}{12} \log \left(\frac{256}{n^{4}}\left(t^{2}+3 n^{2} t+9 n^{4}\right)\right) \\
& +\frac{1}{2} \log \left(\frac{|t|}{3}\right)+\frac{1}{2} \log (2)+1.07 \\
\leq & \frac{1}{4} \log \left(t^{2}+3 n^{2} t+9 n^{4}\right)+\frac{1}{2} \log (|t|)+1.561 \\
\leq & \log (|t|)+\frac{1}{4} \log \left(1+\frac{3 n^{2}}{|t|}+\frac{9 n^{4}}{t^{2}}\right)+1.561 .
\end{aligned}
$$


Finally, if $|t| \geq 100 n^{2}$, we obtain

$$
\widehat{h}\left(\left(0, n^{3}\right)\right) \leq \log (|t|)+1.57 .
$$

Remark. We note that the upper bound of Proposition 18 is independent of $n$. In fact, this bound is not optimal. A numerical analysis suggests that $\widehat{h}\left(\left(0, n^{3}\right)\right)$ should be equivalent to $\frac{1}{2} \log \left(\frac{|t|}{n^{2}}\right)$ as $|t| \rightarrow+\infty$.

By an argument similar to Proposition 18, we obtain the following statement.

Proposition 19. For the case $t=4 k+1, n=4 m$. If $|t| \geq 100 n^{2}$, we have

$$
\widehat{h}\left(\left(0,8 m^{3}\right)\right) \leq \log (|t|)+0.19 .
$$

\section{Main results}

Proposition 20. Suppose that $t \geq \max \left(100 n^{2}, n^{4}\right)$ or $t \leq \min \left(-100 n^{2},-2 n^{4}\right)$. Suppose also that the equation $y^{2}=f(x)$ is a minimal Weierstrass model for $E$ when $\delta$ is squarefree (which occurs if $t \not \equiv 1[4]$ when $4 \mid n$ ). Then the point $\left(0, n^{3}\right)$ is not divisible.

Proof. Assume that $t$ is positive. Let $P$ be a point in $E(\mathbb{Q})-E_{0}(\mathbb{Q})$ and $\ell \geq 2$ such that $\ell P=\left(0, n^{3}\right)$. By Lemma $6, P$ must be an integral point. By Theorem 14 we know that $\widehat{h}(P) \geq \frac{13}{80} \log (t)-\frac{1}{8} \log (n)+0.06$ and by Proposition 18, we have $\widehat{h}\left(\left(0, n^{3}\right)\right) \leq \log (t)+1.57$. We note that $P$ has infinite order and then $\widehat{h}(P) \neq 0$. Since $\widehat{h}$ is quadratic, we have

$$
\begin{aligned}
\ell^{2}=\frac{\widehat{h}\left(\left(0, n^{3}\right)\right)}{\widehat{h}(P)} & \leq \frac{\log (t)+1.57}{\frac{13}{80} \log (t)-\frac{1}{8} \log (n)+0.06} \\
& \leq \frac{80}{13}+\frac{\frac{2}{3} \log (n)+1.21}{\frac{13}{80} \log (t)-\frac{1}{8} \log (n)+0.06} \\
& \leq 8.6
\end{aligned}
$$

thus $\ell \leq 2$. However we have seen at the beginning of Section 4.1 that $\ell$ is odd, hence the point $\left(0, n^{3}\right)$ is not divisible. 
Assume now that $t$ is negative. We have by a similar argument (see Theorem 14 and Proposition 18),

$$
\begin{aligned}
\ell^{2} & \leq \frac{\log (|t|)+1.57}{\frac{3}{16} \log (|t|)-\frac{1}{8} \log (n)} \\
& \leq 5.34+\frac{\frac{2}{3} \log (n)+1.57}{\frac{3}{16} \log (|t|)-\frac{1}{8} \log (n)} \\
& \leq 8.85 .
\end{aligned}
$$

Again we get $\ell \leq 2$ which is impossible. Thus the point $\left(0, n^{3}\right)$ is not divisible.

Using Theorem 17 and Proposition 19, we obtain the following statement.

Proposition 21. We assume $n=4 m$ and $t=4 k+1$, for some $m \in \mathbb{Z}_{>0}$ and $k \in \mathbb{Z}$. If $\delta$ is squarefree, and $t \geq \max \left(100 n^{2}, n^{4}\right)$ or $t \leq \min \left(-100 n^{2},-2 n^{4}\right)$, then the point $\left(0, n^{3}\right)$ is not divisible on $E$.

By combining Propositions 20 and 21 , we obtain the main result.

Theorem 22. Suppose that $t \geq \max \left(100 n^{2}, n^{4}\right)$ or $t \leq \min \left(-100 n^{2},-2 n^{4}\right)$, and $\delta$ is squarefree. Then the point $\left(0, n^{3}\right)$ is not divisible on $E$.

Now, to extend Theorem 22 to the case $|t|<\max \left(100 n^{2}, n^{4}\right)$, we can use the lower bound for the height in [7, Proposition 2.1]: for all $P \in E(\mathbb{Q})$ of infinite order, if $P$ is non-singular modulo $p$ for all prime $p$, we have

$$
\widehat{h}(P)>\frac{1}{12 N^{2}} \log \left|\Delta_{\min }\right|,
$$

where $N$ is the number defined in [7, Theorem 1], and $\Delta_{\min }$ is the minimal discriminant of $E$. Let $C_{E}$ be the lowest common multiple of Tamagawa numbers of $E$, and $P \in E(\mathbb{Q})$ a point of infinite order, the point $C_{E} P$ is non-singular modulo $p$ for all prime $p$ so we have

$$
\widehat{h}(P)>\frac{1}{12 N^{2} C_{E}^{2}} \log \left|\Delta_{\min }\right|
$$

Since $\Delta>0$, we have $N=6$ or $N=8$. Hence we have for all $P \in E(\mathbb{Q})$ of infinite order

$$
\widehat{h}(P)>\frac{1}{768 C_{E}^{2}} \log \left|\Delta_{\min }\right| .
$$

Let us denote by $B_{E}$ this lower bound. So for a given $t$, it suffices to check that $\left(0, n^{3}\right)$ is not equal to $\ell P$ for all $P \in E(\mathbb{Q})$ and for all primes $\ell \leq \sqrt{\frac{\widehat{h}\left(0, n^{3}\right)}{B_{E}}}$. 
We use the following method: if there exist a prime $\ell$ and a point $P \in$ $E(\mathbb{Q})$ such that $\ell P=\left(0, n^{3}\right)$ then for all primes $p \nmid \Delta$, the reduction of $E$ modulo $p$ is an elliptic curve over $\mathbb{F}_{p}$ and $\ell \bar{P}=\overline{\left(0, n^{3}\right)}$, where $\bar{P}$ denote the reduction of the point $P$. Since $p \nmid \Delta$ and the point $P$ is an integral point, the reduction modulo $p$ of $P$ is well-defined and is a point of $E\left(\mathbb{F}_{p}\right)$. If $p$ is a prime number such that $\ell$ divides the exponent of group $E\left(\mathbb{F}_{p}\right)$ denoted by $r_{p}$, we shall have $\frac{r_{p}}{\ell} \overline{\left(0, n^{3}\right)}=O$. So, if we find a prime $p$ such that $\ell \mid r_{p}$ and $\frac{r_{p}}{\ell} \overline{\left(0, n^{3}\right)} \neq O$, there does not exist $P \in E(\mathbb{Q})$ such that $\ell P=\left(0, n^{3}\right)$.

We obtain the following statement.

Theorem 23. If $n \leq 10$ and $\delta$ is squarefree, then the point $\left(0, n^{3}\right)$ is not divisible.

Proof. Assume $n=1$. Theorem 22 implies that if $|t| \geq 100$, the point is not divisible. We also note that the result is true for negative $t$ because $(0,1)$ is the only integer point in $E(\mathbb{Q})-E_{0}(\mathbb{Q})$. For $1 \leq t<100$, it suffices to use the method described above with the help of PARI/GP [12]. For $2 \leq n \leq 10$, we use a similar method. 


\section{References}

[1] Ayad, M. (1992). Points S-entiers des courbes elliptiques. Manuscripta mathematica, 76(1):305-324.

[2] Bettin, S., David, C., and Delaunay, C. (2018). Non-isotrivial elliptic surfaces with non-zero average root number. Journal of Number Theory, 191:1-84.

[3] Cohen, H. (2008). Number theory: Volume I: Tools and diophantine equations, volume 239. Springer Science \& Business Media.

[4] Cohen, H. (2013). A course in computational algebraic number theory, volume 138. Springer Science \& Business Media.

[5] Delaunay, C. and Duquesne, S. (2003). Numerical investigations related to the derivatives of the L-series of certain elliptic curves. Experimental mathematics, 12(3):311-317.

[6] Duquesne, S. (2001). Integral points on elliptic curves defined by simplest cubic fields. Experimental Mathematics, 10(1):91-102.

[7] Krir, M. (2001). À propos de la conjecture de Lang sur la minoration de la hauteur de Néron-Tate pour les courbes elliptiques sur Q. Acta Arith., 100(1):1-16.

[8] Silverman, J. H. (1985). Divisibility of the specialization map for families of elliptic curves. American Journal of Mathematics, 107(3):555-565.

[9] Silverman, J. H. (1988). Computing heights on elliptic curves. Mathematics of computation, 51(183):339-358.

[10] Silverman, J. H. (1990). The difference between the Weil height and the canonical height on elliptic curves. Mathematics of computation, $55(192): 723-743$.

[11] Silverman, J. H. (2013). Advanced topics in the arithmetic of elliptic curves, volume 151. Springer Science \& Business Media.

[12] The PARI Group (2019). PARI/GP version 2.11.2. Univ. Bordeaux. available from http://pari.math.u-bordeaux.fr/.

[13] Washington, L. (1987). Class numbers of the simplest cubic fields. Mathematics of Computation, 48(177):371-384. 


\author{
Valentin Petit \\ valentin.petit@univ-fcomte.fr \\ Laboratoire de mathématiques de Besançon \\ Université Bourgogne Franche-Comté \\ CNRS UMR 6623 \\ 16 , route de Gray \\ 25030 Besançon Cedex \\ France
}

